\title{
PERFIL DE LOMBALGIA EM ACADÊMICOS DE FISIOTERAPIA: RELAÇÃO COM COLCHÃO E DECÚBITO DURANTE O SONO ${ }^{1}$
}

\author{
LOMBALGY PROFILE IN PHYSIOTHERAPY ACADEMICS: \\ RELATION WITH MATTRESS AND DECUBITUS DURING SLEEP
}

\author{
Leonardo Passidonia dos Reis ${ }^{2}$, Jéssica Caroline Cruz Sanches ${ }^{3}$ e \\ Henrique Eduardo Maia Alves ${ }^{4}$
}

\section{RESUMO}

A lombalgia é um dos grandes problemas da saúde pública, que vem aumentando cada vez mais entre jovens estudantes, pode ser definida como um sintoma que afeta a área entre a porção mais baixa do dorso e a prega glútea e apresenta causa multifatorial. Este estudo teve como objetivo verificar a prevalência de lombalgia em acadêmicos de Fisioterapia e relação com decúbito adotado durante o sono e tipo de colchão. Trata-se de um estudo de campo com levantamento de dados, objetivo exploratório de natureza quantitativa. A pesquisa foi desenvolvida em uma instituição de ensino superior privada da região norte do Brasil, no primeiro semestre de 2018, com acadêmicos regularmente matriculados na graduação de Fisioterapia, com aplicação de questionário adaptado e Escala Visual Analógica de dor. Foi observado prevalência de 86,27\% de dor lombar nos acadêmicos de Fisioterapia sem associação significativa com o decúbito adotado durante o sono e tipo de colchão.

Palavras-chave: Modalidades de Fisioterapia; Lombalgia; Colchão.

\section{ABSTRACT}

Lombalgy is an of the major public health problems, which is increasingly growing among young students. It can be defined as a symptom affecting the area between the lower portion of the back and the gluteal fold and has a multifactorial cause. This study aimed to verify the prevalence of low back pain in physiotherapy students and the relationship with decubitus adopted during sleep and type of mattress. This is a field study with data survey, exploratory objective of quantitative nature. The research was developed in a private higher education institution in the northern region of Brazil, in the first semester of 2018, with academics regularly enrolled in the Physiotherapy undergraduate program, with the application of an adapted questionnaire and Visual Analog Pain Scale. A prevalence of $86.27 \%$ of low back pain was observed in students of Physical Therapy without significant association with the position adopted during sleep and type of mattress.

Keywords: Physical Therapy Modalities; back pain; mattress.

\footnotetext{
${ }^{1}$ Tipo de Trabalho: Trabalho de Conclusão de Curso

${ }^{2}$ Fisioterapeuta graduado pelo Centro Universitário Luterano de Palmas. Email:leonardopossidone@gmail.com

${ }^{3}$ Fisioterapeuta graduada pelo Centro Universitário Luterano de Palmas. Email: jessicacarolinecruz@gmail.com

${ }^{4}$ Fisioterapeuta - Mestre em Fisioterapia - Docente no Centro Universitário Luterano de Palmas. Email: henriquemaia@ ceulp.edu.br
} 


\section{INTRODUÇÃO}

A lombalgia é definida como um sintoma que afeta a área entre a porção mais baixa do dorso e a prega glútea, podendo ainda ser irradiada para os membros inferiores, determina um quadro clínico caracterizado por dor, incapacidade de se movimentar e trabalhar (ALMEIDA, 2015).

Atualmente, a dor nas costas em estudantes apresenta alta prevalência, como mostra o estudo de Paananen et al. (2010) onde demonstrou que a ocorrência de dor músculoesquelética é frequente em jovens escolares, motivo de preocupação, principalmente por estar relacionada ao estilo de vida dos jovens, a qual inclui os hábitos posturais adotados durante a vida e as alterações na postura corporal. $\mathrm{Na}$ realidade, as pessoas mais jovens podem sofrer mais vezes de dores mais fortes do que as mais idosas, pois com o envelhecimento a tendência é uma redução na sensibilidade dolorosa. Possui causas multifatoriais como excesso de peso do material escolar e transporte inadequado (ZACHARY et al., 2010; OZGUL, 2012), mobílias inadequadas, atividades de vida diária (AVDs) com posturas incorretas, permanecer sentado por períodos prolongados, sedentarismo, postura incorreta ao dormir, além do colchão inadequado e fatores psicossociais, como, por exemplo, depressão e ansiedade. Posturas inadequadas durante períodos prolongados podem tornar-se hábitos posturais, levando eventualmente o indivíduo a desenvolver alterações posturais e biomecânicas. A boa postura refere-se àquela que a pessoa mantém com o mínimo de esforço muscular, sendo que posturas inadequadas podem aumentar o estresse sobre elementos corporais, e o distribui para estruturas com menor capacidade de suportá-lo, provocando mudança no centro de gravidade, e em consequência, alteração biomecânica e dor. Mostrando assim a importância dos programas educativos que interferem sobre os riscos comportamentais posturais, que podem ser modificados (BENINI; KAROLCZAK, 2010; NOLL; CANDOTTI; VIEIRA, 2012; FURLANETTO; MEDEIROS; CANDOTTI, 2015; NOLL et al., 2016).

A dor lombar atinge grande parte da população mundial, afetando seu bem estar e saúde, elementos fundamentais para a qualidade de vida. Estima que cerca de $80 \%$ da população mundial terão, pelo menos, um episódio de dor na coluna durante a vida (WALKER, 2000). Sendo um importante problema de saúde pública (MONTENEGRO, 2011; NOLL et al., 2016). Este estudo teve como objetivo verificar a prevalência de lombalgia em acadêmicos de Fisioterapia e relação com decúbito adotado durante o sono e tipo de colchão.

\section{METODOLOGIA}

Trata-se de um estudo de campo com levantamento de dados com objetivo exploratório de natureza quantitativa. A pesquisa foi desenvolvida em uma instituição de ensino superior privada da 
região norte do Brasil, no primeiro semestre do ano de 2018, com acadêmicos regularmente matriculados na graduação de Fisioterapia. A coleta dos dados foi realizada através da abordagem direta dos acadêmicos para as devidas explicações sobre os objetivos da pesquisa e após assinatura do Termo de Consentimento Livre e Esclarecido (TCLE) foi realizado aplicação de questionário adaptado de Almeida (2015).

Esse projeto foi submetido à avaliação do Comitê de Ética em pesquisa para apreciação, de acordo com as normas estabelecidas pelo próprio Comitê de Ética e pela Resolução 466/12 do Conselho Nacional da Saúde e aprovado pelo CAAE no 79842817.9.0000.5516.

Foram incluídos acadêmicos matriculados na graduação em Fisioterapia, faixa etária maior ou igual a 18 anos de idade, ambos os sexos e excluídos indivíduos que passaram por procedimento cirúrgico em qualquer região da coluna vertebral, seja lombar, torácica ou cervical.

O Questionário Adaptado de Almeida (2015) organizado com 11 perguntas diretas relacionadas a posição de dormir, tipo de colchão, presença e frequência de dor lombar, prática de exercício físico e uso de medicamentos. Também foi aplicado o instrumento de quantificação da dor, "Escala Visual Analógica (EVA)", um método simples e bastante utilizado, onde é apresentado ao indivíduo uma linha de dez centímetros, que possui em cada extremidade, respectivamente, os números 0 e 10 , onde 0 indica ausência de dor e o 10, representa a pior dor imaginável, o indivíduo situa nesta escala a intensidade da sua dor na região lombar.

Os dados foram transferidos para planilhas de Excel (versão 97-2003), para posteriormente serem exportados e analisados no programa de distribuição livre Epi info 7.1.5.2 ${ }^{\mathrm{TM}}$ (CDC, 2015). Foram realizadas estatísticas descritivas (VIEIRA, 1981) visando apresentar os dados em tabelas, gráficos e frequências absolutas e relativas. Para estudar a associação entre variáveis independentes (sexo e medicamento) e a dor auto referida (Sim/Não) foi utilizado o Teste Exato de Fisher (SOARES; SIQUEIRA, 2002). Para analisar a relação entre as variáveis (idade, posição para dormir e o tipo de colchão) e a dor auto referida (Sim/Não) foram usados testes de Qui-Quadrado (CALLEGARI-JACQUES, 2003). Em todos os testes foi considerado um $\alpha=0,05$ para o Erro Tipo I (nível de significância).

\section{RESULTADOS E DISCUSSÃO}

Participaram da pesquisa 51 acadêmicos de 8 períodos do curso de Fisioterapia $\left(2^{\circ}, 3^{\circ}, 4^{\circ}, 5^{\circ}\right.$, $6^{\circ}, 7^{\circ}, 9^{\circ}$ e $10^{\circ}$ período). Dos 51 acadêmicos, 44 relataram sentir dor na coluna lombar, representando $86,27 \%$ mostrando alta prevalência de dor lombar entre os acadêmicos do curso de Fisioterapia. Desse total 2 são do sexo masculino e 49 do sexo feminino (Tabela 1). A associação entre sexo e dor auto referida não foi significativa pelo Teste Exato de Fisher $(p=0,25)$. 
Tabela 1 - Relação entre as variáveis de interesse e dor auto referida.

\begin{tabular}{|c|c|c|c|c|}
\hline \multirow{2}{*}{\multicolumn{2}{|c|}{ Variável }} & \multicolumn{2}{|c|}{ Dor Auto referida } & \multirow{2}{*}{$\mathbf{P}$} \\
\hline & & $\mathbf{S}$ & $\mathbf{N}$ & \\
\hline \multirow{2}{*}{ Sexo } & Masc & 1 & 1 & $0,25^{*}$ \\
\hline & Femin & 43 & 6 & \\
\hline \multirow{5}{*}{ Idade } & $18+20$ & 8 & 0 & $0,22 * *$ \\
\hline & $20+22$ & 17 & 4 & \\
\hline & $22+24$ & 15 & 1 & \\
\hline & $24+26$ & 2 & 1 & \\
\hline & 26 - 28 & 2 & 1 & \\
\hline \multirow{2}{*}{ Medicamento } & Sim & 13 & 1 & $0,65^{*}$ \\
\hline & Não & 31 & 6 & \\
\hline \multirow{4}{*}{ Posição Dormir } & Decúbito Dorsal & 4 & 0 & $0,79 * *$ \\
\hline & $\begin{array}{c}\text { Decúbito Lateral com } \\
\text { travesseiro MMII }\end{array}$ & 8 & 2 & \\
\hline & $\begin{array}{c}\text { Decúbito Lateral sem } \\
\text { travesseiro MMII }\end{array}$ & 11 & 2 & \\
\hline & Decúbito Ventral & 21 & 3 & \\
\hline \multirow{4}{*}{ Colchão } & Firme & 9 & 0 & $0,07^{* *}$ \\
\hline & Médio Firme & 24 & 3 & \\
\hline & Macio & 8 & 2 & \\
\hline & Muito Macio & 2 & 3 & \\
\hline \multirow{2}{*}{ Atividade física } & Sim & 23 & 4 & $0,88^{* *}$ \\
\hline & Não & 21 & 3 & \\
\hline
\end{tabular}

Teste Exato de Fisher (*) Qui Quadrado (**).

A variável categórica idade (anos) variou entre 18 27 anos. A maior frequência de classe observada foi entre 20 22 anos, com 21 acadêmicos; seguida da classe 22 - 24 anos com 16 representantes. As duas classes de idade maiores (24-26 e 26- 28) apresentaram o menor número de acadêmicos, como pode ser observado na Tabela 1 pelo teste de qui quadrado, não houve associação entre classe de idade (anos) e dor auto referida.

No estudo de Vey, Silva e Lima (2012) realizado com 90 alunos de graduação de diversos cursos, dentre eles Fisioterapia, com média de 24,8 anos, sendo que 82,23\% dos graduandos relataram dores na coluna vertebral, destes $47,9 \%$ relataram dor na região lombar, mostrando a alta prevalência de lombalgia em graduandos, corroborando com os achados desta pesquisa.

Neste estudo observou-se que dos 51 participantes da pesquisa, 49 são do sexo feminino, onde destas 43 acadêmicas relataram sentir dor na coluna lombar, condizendo com o estudo de Neto et al. (2016) que realizaram um estudo com 200 universitários, onde $81 \%$ eram do sexo feminino, deste total $65 \%$ eram graduandos em Fisioterapia e 66\% relataram dor na coluna lombar. O estudo de Noll et al. (2013) também mostra uma maior prevalência de dor lombar no sexo feminino, estima que o resultado está de acordo com o amadurecimento precoce que ocorre no sexo feminino e das características anátomo-funcionais distintas em relação ao sexo masculino. A relação entre dor lombar e gênero não pode ser analisada neste estudo, devido prevalência do sexo feminino na população estudada. 
Dos 51 acadêmicos que participaram da pesquisa 14 tomam medicamentos $(27,45 \%)$ e 37 não fazem uso de alopáticos. Neste quesito não houve associação com sintomas auto referidos de dor $(\mathrm{p}=0,65)$.

Segundo o estudo de Neres et al. (2010) realizado com acadêmicos do curso de Fisioterapia onde 46,9\% dos acadêmicos realizavam o uso de medicamentos sem prescrição e avaliação médica. A automedicação é uma prática universal e perigosa, presente nas mais diversas sociedades e culturas, sendo que as mulheres são as que mais se auto medicam. Pardo et al. (2013) concluíram em estudo realizado com jovens estudantes que $96,20 \%$ se automedicam em algum momento. Devido a facilidade de se conseguir adquirir medicamentos esse número de pessoas que se auto medicam pode aumentar cada vez mais, outro fator que alavanca esse grande número de jovens que se auto medicam é pela influência dos próprios pais e responsáveis ou amigos, em estudo realizado com universitários mostrou que 41,2\% das automedicações eram por orientação de um parente (SCHUELTER-TREVISOL et al., 2011).

Quando analisada a associação entre três categorias de posição para dormir, decúbito dorsal (DD), decúbito lateral (DL) e decúbito ventral (DV) e dor (Sim, Não) não foi encontrada associação significativa pelo teste de qui quadrado $\left(\chi_{0,05 ; 2}^{2}=0,92 ; \mathrm{p}=0,62\right)$.

De acordo com o estudo de Noll et al. (2013) recomenda-se como melhor posição para se dormir o decúbito lateral e o decúbito dorsal. Na pesquisa de Furlanetto, Medeiros e Candotti (2015) o decúbito lateral também foi considerada a posição ideal para se dormir, considerando incorreto o decúbito ventral e o decúbito dorsal, sendo que nessa mesma pesquisa o decúbito lateral apresentou uma maior relação com dor nas costas que os outros decúbitos (ventral e dorsal). Em estudo realizado por Noll et al. (2016) com estudantes, foi observado que os alunos que dormiam em decúbito dorsal obtiveram um menor índice de dor nas costas e os que dormiam em decúbito lateral foram os que mais se queixavam de dor nas costas. Sendo que na atual pesquisa o decúbito dorsal foi o que teve maior índice de dor na coluna lombar dentre os acadêmicos que participaram da pesquisa. O estudo de Desouzart et al. (2014) realizado com acadêmicos, também relaciona um maior número de queixas de dor nas costas pelos acadêmicos que dormiam em decúbito lateral. Furlaneto, Medeiros e Candotti (2015) e Noll et al. (2016) também tiveram o mesmo achado em sua pesquisa. Indo contra o que é dito e indicado por profissionais da saúde, sendo que um grande número de profissionais da saúde indica e incentiva seus pacientes de forma errônea a adotar o decúbito lateral, sendo que não existe evidencia cientifica de que ao adotar o decúbito lateral durante o sono estará prevenindo problemas da coluna lombar.

De acordo com a cartilha da Associação Brasileira de Reabilitação da Coluna (MONTENEGRO, 2011) a posição recomendada para se dormir é o decúbito lateral com travesseiro entre as pernas, ditando o decúbito dorsal como a forma inadequada de se dormir. Pode-se observar que tanto a literatura empírica como os profissionais da área da saúde orientam a população a adotar o decúbito lateral como adequado para uma melhor qualidade do sono e mais indicada como prevenção de alterações na coluna lombar. Apesar deste estudo não ser estatisticamente significativo ao correlacionar o decúbito adotado durante o sono com dor na coluna lombar, 23 acadêmicos relataram dormir em decúbito 
lateral e destes 19 relataram sentir dor na coluna lombar correspondendo a 82,60\%, dos 4 acadêmicos que relataram dormir em decúbito dorsal 100\% sentem dor na coluna lombar, dos 24 estudantes que relataram dormir de decúbito ventral 87,5\% sentem dor na coluna lombar. Pereira et al., (2009) relataram em seu estudo que a postura adequada é aquela que reduz o estresse e relaxa os músculos.

A maciez do colchão utilizado pelos acadêmicos variou entre muito macio e firme. Sendo que a categoria Médio Firme é a mais utilizada (27 acadêmicos), como pode ser observado na Tabela 1. A associação estatística entre dor auto referida e maciez do colchão não foi significativa $(p=0,07)$, como pode ser observado na Tabela 1 .

O colchão deve acomodar as curvaturas da coluna ou as saliências do corpo de modo que mantenha a coluna com o mínimo estresse, e também indica que o colchão muito macio comumente não oferece o apoio adequado para a acomodação da coluna vertebral (MONTENEGRO, 2011). Machado (2008) recomenda em sua pesquisa a utilização de colchão médio firme, afirma que um colchão muito macio permite que o corpo afunde causando desequilíbrio das curvaturas da coluna. Almeida (2015) também indica em seu estudo o colchão médio firme como o melhor para evitar dor na coluna vertebral.

O estudo realizado por Jacobson (2009) com 59 pessoas, chegou à conclusão que o colchão médio firme resultou em uma melhor qualidade do sono com diferença significativa em relação a dor nas costas. Podemos observar no presente estudo que o colchão médio firme foi mais utilizado pelos acadêmicos (27) sendo que 88,88\% sentem dor na coluna lombar, 9 relataram dormir em colchão firme destes $100 \%$ relatam sentir dor na coluna lombar, 10 em colchão macio destes $80 \%$ sentem dor na coluna lombar, e um estudante relatou dormir em colchão muito macio e não sente dor na coluna lombar.

Verificando os sintomas de dor (Sim, Não) e atividade física (Sim, Não), mediante o teste de qui quadrado, não houve associação entre estas duas variáveis de interesse $\left(\chi_{\text {yates } 0,05}^{2}=0,25 ; p=0,88\right)$, onde dos 44 acadêmicos que relatam dor 52,27\% praticam atividade física e dos 7 acadêmicos que relatam não sentir dor $57,14 \%$ praticam atividade física.

A relação entre prática de atividade física e lombalgia ainda não está totalmente elucidada (OLIVEIRA; SALGUEIRO; ALFIERI, 2015). Segundo o trabalho realizado por Noll et al., (2016) não há relação entre a prática de atividade física e dor na coluna lombar. Em relação à prática de atividade física e lombalgia não houve relação, sendo que dos 51 participantes da pesquisa 27 relataram praticar atividade física e destes 85,18\% sentem dor na lombar, e dos 24 estudantes que disseram não praticar atividade física $87,5 \%$ sentem dor na lombar.

Ao considerar o tempo em semanas dos sintomas, pode ser verificado na Figura 1 que $61 \%$ dos acadêmicos que participaram da pesquisa sentem dor há mais de 12 semanas, $21 \%$ dos participantes sentem dor há menos de 6 semanas e 18\% sentem dor entre 6 e 12 semanas. 
Figura 1 - Relação de dor e tempo em semanas do sintoma.

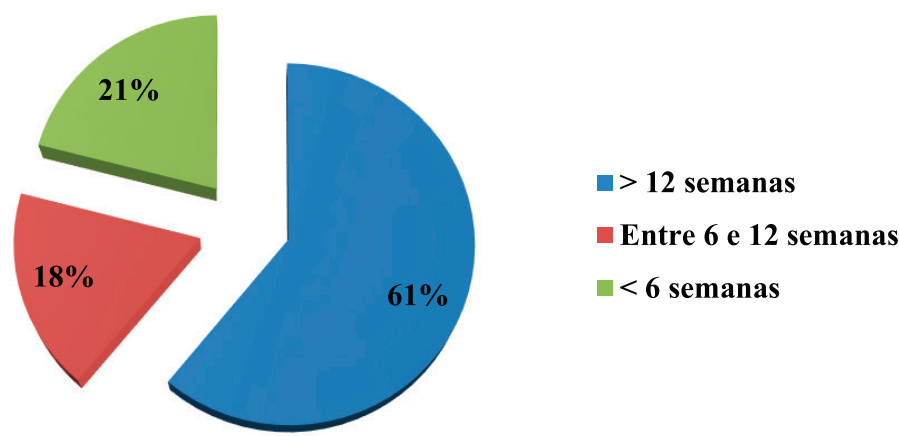

Segundo Noll et al. (2016) 55,7\% dos estudantes analisados disseram sentir dor nas costas nos últimos 3 meses. De acordo com Almeida (2015) a dor pode ser classificada em aguda, com duração menor que quatro semanas; subaguda, com duração entre a quatro semanas e 12 semanas e, crônica, com duração acima de 12 semanas, onde o mesmo relata em seu estudo que cerca de $10 \%$ das pessoas sofrem de dor crônica.

Em relação ao período do dia com maior intensidade dos sintomas, dos 44 acadêmicos que relataram sentir dor na coluna, em sua maioria sentem a dor, representando 59,09\% sentem no período da noite $(\mathrm{N})$ e $25 \%$ dos participantes da pesquisa sentem dor no período da tarde. Três categorias $(\mathrm{M} / \mathrm{N} ; \mathrm{M} / \mathrm{T}$ e $\mathrm{T} / \mathrm{N})$ apresentaram apenas 1 acadêmico cada.

Figura 2 - Relação da dor com o período do dia de maior intensidade dos sintomas.

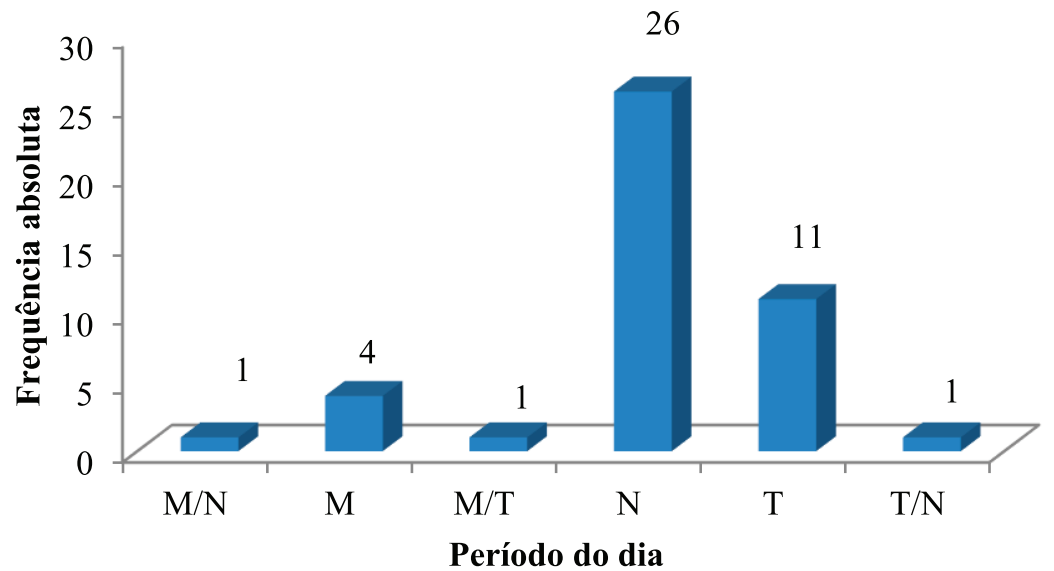

M: manhã; T: tarde; N: noite.

Dos acadêmicos que relataram sentir dor na coluna lombar, em relação ao período do dia, a maioria relatou o período da noite, podendo ser explicado pelo fato dos acadêmicos passarem muitas horas do dia adotando posturas inadequadas, por permanecer muito tempo sentado ou devido adotarem posturas inadequadas durante suas aulas e atividades. Segundo o estudo de Coelho (2017) a 
dor na coluna lombar relaciona-se, muitas vezes à permanência constante na postura sentada e inadequada, o que leva a um aumento de pressão no disco intervertebral e sobrecarga da musculatura paravertebral lombar.

Na distribuição da escala visual analógica de dor (EVA), dos 51 acadêmicos que participaram da pesquisa, 29,41\% relataram que sentem dor 4 , sendo que a média de dor lombar dos acadêmicos participantes da pesquisa foi de 3,50 .

Tabela 2 - Distribuição da EVA de dor lombar nos acadêmicos.

\begin{tabular}{|ccc|}
\hline EVA Lombar & Número de acadêmicos & \% Porcentagem \\
\hline $\mathbf{0}$ & 9 & 17,6 \\
$\mathbf{1}$ & 3 & 5,88 \\
$\mathbf{2}$ & 6 & 11,76 \\
$\mathbf{3}$ & 4 & 7,84 \\
\hline $\mathbf{4}$ & 15 & 29,41 \\
$\mathbf{5}$ & 5 & 9,8 \\
$\mathbf{6}$ & 3 & 5,88 \\
$\mathbf{7}$ & 0 & 0 \\
$\mathbf{8}$ & 5 & 9,8 \\
$\mathbf{9}$ & 1 & 1,96 \\
\hline $\mathbf{1 0}$ & 0 & 0 \\
TOTAL & 51 & 100 \\
\hline Média de dor (EVA): $\mathbf{3 , 5 0}$ & & \\
\hline
\end{tabular}

\section{CONCLUSÃO}

Pode-se verificar que dentre os acadêmicos do curso de Fisioterapia de instituição de ensino superior privada da região norte do Brasil a lombalgia possui alta prevalência. Foi observado que o período do dia com maior intensidade de dor lombar foi no período da noite e que a maioria se caracteriza como lombalgia crônica. Não existe relação entre o decúbito adotado durante o sono e tipo de colchão com dor na coluna lombar.

\section{REFERÊNCIAS}

ADORNO, Marta Lúcia Guimarães Resende; BRASIL-NETO, Joaquim Pereira. Avaliação da qualidade de vida com o instrumento SF-36 em lombalgia crônica. Acta ortop. bras, v. 21, n. 4, p. 202-207, 2013. Disponível em: https://bit.ly/35N5LJ7. Acesso em: 22 de março de 2017

ALMEIDA, Gustavo Coutinho Desouzart de. Efeitos de uma intervenção postural durante o sono, na percepção da dor na coluna vertebral, na qualidade de vida e na qualidade do sono. 2015. $244 \mathrm{f}$. Tese (Doutorado) - Curso de Comportamento Motor, Universidade de Lisboa Faculdade de Motricidade Humana, Lisboa, 2015. 
ANDRADE, Karen Fernandes. Estresse e desordem temporomandibular (DTM) - incidência e relação em docentes de nível superior na cidade de Palmas - Tocantins. 2009. 131 f. Dissertação (Mestrado) - Curso de Ciência da Educação, Universidade Lusófona de Humanidades e Tecnologias, Lisboa, 2009.

BENINI, Juliana; KAROLCZAK, Ana Paula Barcellos. Benefícios de um programa de educação postural para alunos de uma escola municipal de Garibaldi, RS. Fisioterapia e Pesquisa, São Paulo, v. 17, n. 4, p. 346-351, 2010.

CALLEGARI-JACQUES, Sidia M. Bioestatística princípios e aplicações. 1. reimp. Porto Alegre: ARTMED, 2003.

CENTERS FOR DISEANE CONTROL AND PREVENTION. Epi Info 7.1.5.2 ${ }^{\mathrm{TM}}$ for Windows: a word processing, database and statistics program for epidemiology version 7.1.5.2. Atlanta: CDC, 2015.

COELHO, Carlos Jhone. Prevalence, frequency and factors associated to back pain in a group of university students. Ciências da Saúde, Fortaleza-ce, v. 3, n. 1, p. 9-15, 2017.

DA SILVA, Renê Gonçalves; MEJIA, Dayana Priscila Maia. A Utilização do Isostretching no tratamento da Hérnia de Disco Lombar. p. 1-14. Disponível em: https://bit.ly/2zdpkOk. Acesso em: 09 abr. de 2017.

\section{DESOUZART, Gustavo et al. HumanBody-Sleep System Interaction in Residence for University}

Students: Evaluation of Interaction Patterns Using a System to Capture Video and Software with Observation of Postural Behaviors During Sleep. Proceedings Of The 5th International Conference On Applied Human Factors And Ergonomics. 2014, Kraków, Poland, p. 1-11, July 2014. Disponível em: https://bit.ly/2L7OKQ2. Acesso em: 24 mar. 2017.

FINIMUNDI, Márcia et al. Validação da escala de ritmo circadiano - ciclo vigília/sono para adolescentes. Revista Paulista de Pediatria, [s.1.], v. 30, n. 3, p. 409-414, set. 2012. FapUNIFESP (SciELO). Disponível em: https://bit.ly/2YDULvZ.

FURLANETTO, Tássia Silveira; MEDEIROS, Fernanda da Silva; CANDOTTI, Cláudia Tarragô. Prevalência de dor nas costas e hábitos posturais inadequados em escolares do Ensino Fundamental do Colégio de Aplicação da UFRGS. Cadernos do Aplicação, Porto Alegre, v. 28, n. 27, p. 99-108, 2015. 
GALDINO, Angélica de Souza; SOARES, Marcelo Márcio. Mobiliário hospitalar sob a ótica da ergonomia o caso dos sistemas de descanso para acompanhantes pediátricos. Ação Ergonômica, Paraiba, v. 1, n. 2, p. 118-128, 2001.

GUERRA, Luciana Aparecida; CANO, Maria Aparecida Tedeschi; ZAIA, Jose Eduardo. Interferência da educação postural sobre algia na coluna vertebral e a postura corporal com alunos da $4^{\mathrm{a}}$ série da rede pública de ensino fundamental. Efdeportes Digital, Buenos Aires, v. 123, n. 13, p. 1-5, 2008. Disponível em: https://bit.ly/2L4OzoT. Acesso em: 24 mar. 2017.

JACOBSON, Bert H.; BOOLANI, Ali; SMITH, Doug B..Changes in back pain, sleep quality, and perceived stress after introduction of new bedding systems. J ChiroprMed, v. 8, n. 1, p. 1-8, mar. 2009. Disponivel em: https://bit.ly/2SGTAYQ. Acesso em 28 de abril de 2017.

MACHADO, Marcia Jascov Mascarenhas. Postura Corporal. Curitiba: Secretaria de Estado da Educação do Paraná 2008. p. 1-20.Disponivel em: https://bit.ly/2SGzlvA. Acesso em 22 de março de 2017.

MONTENEGRO, Helder. Associação Brasileira de Reabilitação de Coluna. Cartilha Dr Coluna. 2011. p. 1-36. Disponivel em: https://bit.ly/2YEaVFz. Acesso em: 07 mar 2017.

NERES, Bruna SI et al. Prevalência da automedicação em acadêmicos de fisioterapia de uma Instituição de Ensino Superior de Teresina. Conscientiae Saúde, v. 9, n. 1, 2010.

NOLL, Matias; CANDOTTI, Cláudia Tarragô; VIEIRA, Adriane. Escola postural: revisão sistemática dos programas desenvolvidos para escolares no Brasil. Ensaios Movimento, Porto Alegre, v. 18, n. 4, p. 265-291, dez. 2012. Disponível em: https://bit.ly/2Wa1r3q. Acesso em: 25 mar. 2017.

NOLL, Matias et al. Dor nas costas, hábitos posturais e comportamentais de escolares de rede municipal de ensino de Teutônia, RS. Journal of Human Growth and Development. Porto Alegre. v. 23, n. 2, p. 87-92, 2013.

NOLL, Matias et al. Fatores de risco associados à intensidade de dor nas costas em escolares do município de Teutônia (RS). Ciência do Esporte, Ceres- Go, v. 2, n. 38, p. 124-131, 2016.

OLIVEIRA, Janaina Gonçalves; SALGUEIRO, Marcia Maria Hernandes de Abreu de Oliveira; ALFIERI, Fábio Marcon. Lombalgia e Estilo de vida. Journal of Health Sciences, São Paulo, v. 16, n. 4, p. 341-344, 2014. 
OZGUL, Bahar et al. Effects of unilateral backpack carriage on biomechanics of gait in adolescents: a kinematic analysis. Acta Orthop Traumatol Turc, v. 4, n. 46, p. 269-274, 2012.

PAANANEN, Markuset al. Risk factors for persistence of multiple musculoskeletal pains in adolescence: A 2-year follow-up study. European Journal of Pain, Chichester, v. 10, p. 1026-1032, 2010.

PARDO, Inês Maria Crespo Gutierres et al. Automedicação: prática frequente na adolescência? Estudo em uma amostra de estudantes do ensino médio de Sorocaba. Ciênc. Méd. Sorocaba, Sorocaba-SP, v. 15, n. 2, p. 11-17, 13 jun. 2012.

PEREIRA, Fernanda Cristina et al. Relação da postura adotada para dormir e a queixa de lombalgia. p. 1-5. V Epcc, Maringá, 27 out. 2009.

REBOLHO, Marilia Christina Tenorio. Efeitos da educação postural nas mudanças de hábitos em escolares das $1^{\mathrm{a}}$ a $4^{\mathrm{a}}$ series do ensino fundamental. 2005. 109 f. Dissertação (Mestrado) - Curso de Mestre em Ciências, Fisiopatologia Experimental, Faculdade de Medicina da Universidade de São Paulo, São Paulo, 2005. Disponível em: https://bit.ly/2Wai0w3. Acesso em: 25 mar. 2017

SCHUELTER-TREVISOL, Fabiana et al. Automedicação em universitários. Rev Bras Clin Med, São Paulo, v. 6, n. 9, p.414-17, Nov. 2011. Disponivel em: https://bit.ly/3dkKOrm. Acesso em 12 mar. 2020

SILVA, Priscila Rejane Lima da. Prevalência de hábitos posturais adequados e de dor nas costas em escolares do ensino médio da rede estadual do município de Esteio-RS. 2013. 35 f. TCC (Graduação) - Curso de Educação Fisica, Universidade Federal do Rio Grande do Sul, Porto Alegre, 2013. Disponível em: https://bit.ly/2L3r3Iz. Acesso em: 29 abr. 2017.

SOCIEDADE BRASILEIRA DE REUMATOLOGIA. Coluna: cartilha para paciente. Comissão de Coluna Vertebral, 2011. p. 1-19.

VEY, Ana Paula Ziegler; SILVA, Alyssa Conte da; LIMA, Francisco Solano Trindade de. Análise de dor nas costas em estudantes de graduação. Disciplinarum Scientia: Série: Ciências da Saúde, Santa Maria, v. 14, n. 2, p. 217-225, 11 jan. 2013. Disponível em: https://bit.ly/2YINV8n. Acesso em: 1 abr. 2017.

VIEIRA, Sonia. Introdução à Bioestatística. Rio de Janeiro: Campus, 1981. 
WALKER, Bruce. The Prevalence of Low Back Pain: A Systematic Review of the Literature from 1966 to 1998. Journal of Spinal Disorders, Australia, v. 13, n. 3, p. 205-217, jun.2000. Disponivel em: https://bit.ly/2W8FRMB. Acesso em 10 fev. 2019

ZACHARY, Heuscher et al. The association of self-reported backpack use and backpack weight with low back pain among college students. J Manipulative Physiol Ther, v. 33, n. 6, p. 432-437, ago. 2010 